\begin{tabular}{|l|c|c|c|r|}
\hline $\begin{array}{l}\text { Cuadernos de Investigación Geográfica } \\
\text { Geographical Research Letters }\end{array}$ & 2018 & N $^{\circ} 44(1)$ & pp. 349-375 & $\begin{array}{r}\text { ISSN 0211-6820 } \\
\text { eISSN 1697-9540 }\end{array}$ \\
\hline
\end{tabular}

\title{
EL CONSENSO SOBRE EL ORIGEN HUMANO DEL CAMBIO DEL CLIMA NO HA SIDO DEMOSTRADO AÚN
}

\author{
J.C. GONZÁLEZ-HIDALGO
}

Department of Geography and Land Management, University of Zaragoza, 50009 Zaragoza, Spain.

\begin{abstract}
RESUMEN. Durante las últimas tres décadas hemos asistido a la configuración de dos nuevos paradigmas. El primero de ellos en el ámbito científico es el llamado cambio del clima, y el segundo que podríamos denominar paradigma científico-social, y que se refiere al primero, el consenso sobre el origen humano del cambio del clima a partir de las emisiones por la quema de combustibles fósiles.

En el presente trabajo el autor revisa los principales textos que han dado lugar al segundo después de una breve presentación del primero siguiendo las principales conclusiones de los informes del Panel Intergubernamental del Cambio del Clima (IPCC). No es objeto de discusión o debate de este trabajo el cambio del clima ni su origen, pero sí lo es cómo se ha llegado a un consenso sobre su origen que es aparente, porque en realidad los textos que lo proponen no lo han demostrado.
\end{abstract}

La revisión de los trabajos más citados en todos los ámbitos, científicos, políticos, culturales, medios de comunicación etc., refleja que la cifra manejada de consenso en torno al $97 \%$ no se ajusta a los datos que los textos presentan, surge de un tratamiento parcial y sesgado de la información, y se refiere siempre a la opinión de un reducido número de personas. La revisión de los datos publicados sugiere con todas las cautelas que una cifra más acorde a la realidad estaría en torno al 50\%.

Las temperaturas han aumentado desde que se tienen registros en observatorios, y no hace falta ningún consenso para afirmar esta realidad empírica. Pero sostener por una simple cuenta de mayorías o por el criterio de autoridad que la causa predominante es la emisión de gases derivados de la quema de combustibles fósiles no es científicamente lícito, pues nunca una mayoría ha sido prueba de verdad.

Consensus on human driving factors of climate change has not been demonstrated yet

ABSTRACT. During the last three decades two new paradigms have emerged. The first one in the called "Climate Change", and the second, that could be named scientific-social paradigm, and referred to the previous, is the consensus that climate change has been produced by human emissions. 
In this paper, after a brief presentation of the first paradigm following the main conclusions of IPCC, the author reviews the main documents from which the second one has been stated. It is not the aim of this paper to argue or discuss the climate change and its attribution, but how the consensus has been achieved, because the consensus on the attribution of climate change is apparent, since it cannot be concluded from the aforementioned text.

The review of the most well-known and quoted papers in scientific, political, cultural, and mass media, shows that $97 \%$ magnitude of consensus is not related to the original data, it has been calculated from partial slanted and biased information, and is referred to the opinion of a small number of people. With caution, the most real agreement from the data published until present should be approximately $50 \%$.

Temperature has risen from the beginning of surface observations, but there is not necessary any consensus to support this empirical fact. Meanwhile, to attribute that greenhouse gases emission is the most relevant factor based on majority or authority principles is not scientifically correct, because no one polling has been proof of true.

Palabras clave: cambio del clima, consenso, ciencia, mayorías, postverdad.

Key words: climate change, consensus, science, majority, post-truth.

Correspondencia: José Carlos González-Hidalgo, Departamento de Geografía y Ordenación del Territorio, Universidad de Zaragoza, 50009 Zaragoza, España. E-mail: jcgh@unizar.es

"...we should recognise that we are dealing with a coupled nonlinear chaotic system, and therefore that the long-term prediction of future climate states is not possible." IPCC AR4 WG1

"Seeker (scientific) after truth had an obligation not to believe any consensus, however well established: instead, it was his duty to check for himself, using his own hard-won knowledge and skill. For the road to truth, was long and hard but that is the road we must follow." Abu Ali Ibn al-Hassan Ibn al-Hussain Ibn alHaytham, Matemático del siglo XI

\section{Introducción. ¿Hablamos de lo mismo cuando hablamos del cambio del clima?}

En torno a 1988 la ONU auspició la formación de un organismo intergubernamental encargado de realizar informes sobre el estado del clima y su evolución. Desde entonces ha elaborado cinco cuya estructura se organiza en diversos documentos a partir del 
denominado informe científico (Working Group I, The Physical Science Basis) entre los que destaca el denominado Summary for Policy Maker, algo así como el resumen para los gestores, que compila resumidamente la información más relevante, y que suele acaparar finalmente los titulares. Con independencia de cualquier otra consideración hay un hecho que debe subrayarse: las conclusiones del documento Summary for Policy Maker se adoptan por consenso en reuniones en las que los textos de los científicos se corrigen, modifican, rechazan o matizan por representantes políticos, en un ejercicio de creación de verdad científica bastante alejado de lo que deben ser los criterios en ciencia.

La definición de cambio del clima hubo de esperar al tercer informe del IPCC del año 2001, en cuyo glosario del Apéndice 1 se afirmaba "Climate change refers to a statistically significant variation in either the mean state of the climate or in its variability, persisting for an extended period (typically decades or longer). Climate change may be due to natural internal processes or external forcings, or to persistent anthropogenic changes in the composition of the atmosphere or in land use". Mantengo el original para evitar equívocos, y acorde el mismo documento se puede deducir que el cambio del clima puede ser originado por factores naturales y humanos. Sin embargo, el documento denominado Framework Convention on Climate Change (UNFCCC), definió en su Art. 1 el cambio del clima como sigue: "a change of climate which is attributed directly or indirectly to human activity that alters the composition of the global atmosphere and which is in addition to natural climate variability observed over comparable time periods". Es decir diferenciando entre cambio del clima, de origen humano, y variabilidad climática, de origen natural. En suma, existen dos definiciones en los documentos de ONU que llevan a la confusión, porque la segunda ha sido la aceptada comúnmente por la comunidad de gestores, también por numerosos científicos, y la que domina socialmente los medios de comunicación. Por otro lado convendría separar tres expresiones que acaban siendo empleadas como sinónimo y no lo son: cambio del clima, calentamiento global, y calentamiento global originado por la emisión de gases con efecto invernadero. Es a esta tercera expresión a la que nos referiremos en adelante.

Desde un punto de vista científico el cambio del clima tal como se está formulando en la actualidad es la hipótesis planteada en los sucesivos informes del IPCC, que ha sido sintetizada por Philip Lloyd (Cape Peninsula University of Technology) y Legates et al. (2013b), de la manera siguiente:

(i) El contenido de $\mathrm{CO}_{2}$ en la atmósfera ha aumentado exponencialmente.

(ii) Dicho aumento es paralelo a la quema de combustibles fósiles, de manera que parece razonable aceptar que su empleo es parejo al aumento de $\mathrm{CO}_{2}$.

(iii) $\mathrm{El} \mathrm{CO}_{2}$ absorbe longitudes de onda en torno a $15 \mu \mathrm{m}$. El $\mathrm{CO}_{2}$ presente en la atmósfera es capaz de hacerlo y posteriormente puede re-emitir parte de esta radiación hacia la superficie.

(iv) En consecuencia, el esperado aumento de energía en la baja troposfera puede originar cambios a largo plazo en el clima caracterizados por: un aumento de 
la temperatura superficial y de los océanos, la frecuencia o magnitud de los eventos extremos, la reducción de la superficie helada etc.

(v) El mayor peligro de estos cambios es el aumento del nivel marino y la inundación de zonas costeras muy pobladas en la actualidad.

En la hipótesis, las tres primeras premisas no ofrecen dudas y se han confirmado: hay más $\mathrm{CO}_{2}$ en la atmósfera que en el inicio de la revolución industrial, cuya procedencia es indiscutible por la quema de combustibles fósiles, y la física y química explican bien su capacidad de absorción y reemisión. De las dos finales existen numerosos estudios que plantean dudas, discrepancias e incertidumbres, entre las que se pueden citar la saturación de las capas bajas de la atmósfera de $\mathrm{CO}_{2}$ y la magnitud de la sensibilidad del clima (Harde, 2014), el retardo en la relación temporal entre el $\mathrm{CO}_{2}$ y las temperaturas (Petit et al., 1999), la discusión sobre la existencia de periodos más cálidos que el actual en época histórica (Luening y Vahrenholt, 2017), la ausencia de aceleramiento en el aumento del nivel marino respecto al siglo XIX (Houston y Dean, 2011), o las incertidumbres de las previsiones de los modelos (Idso et al., 2015; Christy, 2016; Curry, 2015, 2017). En Rapp (2014) y en Idso et al. (2015) se pueden consultar dos revisiones detalladas de los aspectos comentados

Con el tiempo, la hipótesis se ha convertido en el paradigma científico dominante y en un auténtico dogma de la ortodoxia científica, política, social y cultural, especialmente a partir del informe del IPCC de 2001 y el gráfico denominado "palo de hockey", una reconstrucción de la evolución de las temperaturas de los últimos mil años en la que desaparecieron tanto el periodo denominado Óptimo Medieval como la Pequeña Edad de Hielo. Una ortodoxia basada en el consenso, que adquiere legitimidad en proporción creciente a las instituciones y personas que se suman a ella, "fundada en las simulaciones por ordenador, reemplazando las tesis y las observaciones, y en la que los gobiernos han terminado por determinar la naturaleza de la creatividad científica" (Lindzen, 2012; también citado por Rapp, 2014, p. 254-256).

En este trabajo se analizan los principales documentos que se han publicado en los 15 últimos años sobre el denominado consenso científico referido al cambio del clima originado por la intervención humana a través de la emisión de gases de efecto invernadero, los cuales han servido en muchas ocasiones para apoyar las tesis del IPCC, revisando los datos aportados y contrastando las principales críticas recibidas. Muchos de ellos han sido refutados in extenso por diferentes autores no necesariamente partidarios de las tesis contrarias, con argumentos variados que no se expondrán detalladamente en el presente texto remitiendo a las críticas originales. Nuestro objetivo es mostrar cómo se han calculado las cifras de consenso, en su caso contrastarlas con las de las críticas recibidas, y cuando fuera posible recalcularlas.

\section{Estudios sobre el consenso}

\subsection{Beyond the ivory tower: the scientific consensus on climate (Oreskes, 2004)}

El primer trabajo en el que se citó un consenso sobre la naturaleza humana del cambio del clima es el firmado por Orekes (2004), quien clasificó 928 resúmenes 
extraídos de la base de datos ISI del periodo 1993-2003 en distintas categorías. A partir de su clasificación afirmó que el 75\% de aquellos estaba conforme con el aumento de las temperaturas por efecto de la intervención humana; de modo notable además indicó que ninguno estaba en contra de dicho aserto, quedando simplemente como no contrarios, lo que daría realmente unanimidad en las respuestas. El estudio ha sido rebatido por Peiser (2005), Shulte (2008) y Legates et al. (2013a y b), entre otros.

Peiser (2005) realizó una búsqueda, con los mismos criterios y en el mismo periodo que Oreskes, identificó 1117 trabajos en las mismas fuentes (si bien la suma de sus parciales es de 1126), y tras su inspección afirmó que solo 13 se posicionaban a favor de la tesis mantenida por Oreskes en su clasificación. Este análisis hacía variar el porcentaje inicial del consenso a un minúsculo 1,2\% (13/1117). La disparidad de estos resultados es tan abrumadora que necesita alguna explicación.

Oreskes clasificó como textos favorables a su tesis todos los que trataban sobre eventuales impactos y medidas mitigadoras (clases 2 y 3) además de aquellos que suponían una postura claramente favorable a los efectos humanos sobre el clima (clase 1); si en el recuento de Peiser (2005) se agregan las categorías 1 y 2 como "favorables" el total pasa a ser 335, que sobre el total identificado de 1117 producen un 29,8\% de consenso. La cifra nunca ha sido rebatida y su carta a Nature no fue publicada.

Poco después Schulte (2008) estudió el periodo 2003-2007 empleando el mismo protocolo de búsqueda que Oreskes. En su análisis detectó 539 textos de los que solamente 38 eran clasificables en la categoría 1 (favorables) y 244 más en los que se podía aceptar una atribución implícita que respecto al total de 539 suponen un 52,3\% (282/539; la cifra de $45 \%$ que ofrece Schulte se refiere al grupo 2 de atribución implícita). Notablemente un $6 \%$ de textos en la revisión de Schulte rechazaba explícitamente el consenso, que sumados a los que se posicionaban implícitamente contrarios ascenderían a un 7,3\%. El trabajo de Schulte (2008), admitiendo el método y clasificación de Oreskes, en todo caso sugeriría que el consenso habría descendido. En la Tabla 1 se resume la interpretación de Oreskes y las evaluaciones alternativas. Entre otros textos, Legates et al. (2013b) también han criticado este método de asignación así como el cálculo del consenso, cuyo concepto no queda claro.

Tabla 1. Comparación de porcentajes del consenso entre Oreskes y las revisiones de Peiser y Schulte. Porcentajes estimados a partir de los datos indicados por los autores.

\begin{tabular}{|c|c|c|c|c|c|c|}
\hline $\begin{array}{c}\text { Oreskes } \\
\text { Abstract }\end{array}$ & Oreskes & $\begin{array}{c}\text { Peiser } \\
\text { Abstract }\end{array}$ & $\begin{array}{c}\text { Peiser } \\
\text { Favorables } \\
\text { (categoría 1) }\end{array}$ & $\begin{array}{c}\text { Pieser } \\
\text { Favorables } \\
\text { categorías 1-2 }\end{array}$ & $\begin{array}{c}\text { Schulte } \\
\text { Abstract }\end{array}$ & $\begin{array}{c}\text { Schulte } \\
\text { Favorables }\end{array}$ \\
\hline 928 & $75 \%$ & 1117 & $13(1,2 \%)$ & $335(29,8 \%)$ & 539 & $282(52,3 \%)$ \\
\hline
\end{tabular}

\subsection{Examining the scientific consensus on climate change (por Doran y Zimmerman, 2009)}

Doran y Zimmerman (2009) en el ámbito de la tesis máster del primer autor realizaron una encuesta a 10257 científicos procedentes del ámbito académico y gubernamental, 
mayoritariamente de EEUU, y publicaron sus resultados finales en la revista EOS. En total recibieron 3146 respuestas, de las que solamente un 5\% se declaró climatólogo (en torno a 150 personas). El porcentaje citado de consenso a su primera pregunta ("¿las temperaturas respecto a 1800 han ascendido, descendido o se han mantenido?”) fue del $90 \%$, y un $82 \%$ manifestó conformidad a la segunda pregunta acerca de si la actividad humana es un factor significativo en el cambio de las temperaturas. En el texto además se realiza un filtrado de expertos según el número de publicaciones y se presenta un consenso final del $96,2 \%$ y $97,4 \%$ a las dos preguntas.

El artículo recibió inmediatamente duras críticas por su concepción, planteamiento y métodos en cartas al editor de la revista (Granqvist, 2010; Helsdon, 2009), porque la primera pregunta lleva implícita una respuesta obvia (afirmación) y no se deduce nada de ella, y la segunda no especifica de qué manera o con qué intensidad e importancia el efecto de las actividades humanas puede alterar las temperaturas. Pero sin duda la crítica más dura recibida por este trabajo es la del informe de la asociación de meteorólogos canadienses retirados Friends of Science (2014), quienes observan que el consenso de expertos anunciado se debe a los 76 y 75 climatólogos que contestaron afirmativamente a ambas preguntas sobre el total de climatólogos que respondieron a las mismas, respectivamente 79 y 77 . Es decir, si se relaciona las respuestas positivas de 76 y 75 climatólogos a ambas preguntas con el total de la encuesta (3146) el valor de consenso de dichos expertos se reduce a un $2,4 \%$ y $2,3 \%$ en ambas preguntas. Tol (2016) muestra una revisión de los datos originales con un resumen de los porcentajes alcanzados por especialidad profesional, tanto en el caso de revisión de publicaciones como de número de autores, que puede ayudar a completar la idea de consenso que se puede deducir de este trabajo, si bien algunas cifras no coinciden con las del original.

Es decir, el porcentaje de consenso de los expertos que Doran y Zimmerman (2009) presentan procede de 75 personas relacionadas con la climatología, si bien no incluyen en su concepción de expertos la categoría de meteorólogos, 36 en total con un porcentaje de consenso de $64 \%$. Por último la cifra indicada en el original de climatólogos encuestados (5\% del total) también pudiera ser la cifra de comparación con las 75 respuestas afirmativas, llevando entonces a un $50 \%$ de consenso de los climatólogos expertos (75/150), en todo caso muy alejada del pretendido $97 \%$ cercano a la unanimidad con el que este trabajo es citado (Tabla 2).

Tabla 2. Porcentaje de consenso según Doran y Zimmermann, y sugerido por Friends of Science.

\begin{tabular}{|c|c|c|c|c|c|c|}
\hline \multirow{2}{*}{$\begin{array}{c}\text { Encuestas } \\
\text { totales }\end{array}$} & \multicolumn{3}{|c|}{ Doran y Zimmerman } & \multicolumn{2}{c|}{ Friends of Science } \\
\cline { 2 - 7 } & Favorables & Global \% & $\begin{array}{c}\text { Climatólogos } \\
\text { Favorables }\end{array}$ & $\%$ & $\begin{array}{c}\text { Climatólogos } \\
\text { Favorables }\end{array}$ & $\%$ \\
\hline 3146 & Pregunta 1 & $90 \%$ & $76 / 79$ & $96,2 \%$ & $76 / 3146$ & $2,4 \%$ \\
\hline & Pregunta 2 & $82 \%$ & $75 / 77$ & $97,4 \%$ & $75 / 3146$ & $2,3 \%$ \\
\hline
\end{tabular}

En resumen, el trabajo ofrece unos resultados sumamente insatisfactorios a partir de unas preguntas de las que no se puede deducir ningún tipo de conclusión clara relacionada realmente con lo que se quiere tratar; también porque el modo en que se 
presentan y analizan los datos indica que el número de personas expertas relacionadas con la climatología es muy escaso, y por último porque su selección no tiene por qué ser la más adecuada. Sus conclusiones podrían haberse escrito de otro modo: "75 climatólogos sobre un total de 77 seleccionados están conformes con que el efecto del hombre en el clima no es cero", o de esta otra manera "solamente el 2,3\% de climatólogos de un total de 3146 científicos encuestados, afirman que el hombre ejerce un efecto indeterminado sobre el clima".

\subsection{Expert credibility in climate change (por Anderegg et al., 2010)}

Anderegg et al. (2010) clasificaron un conjunto de 1372 investigadores a través de sus publicaciones y encontraron un consenso entre el 97-98\% sobre la naturaleza humana del cambio del clima acorde con la definición del IPCC. En realidad el porcentaje que expresan como conclusión en su resumen no se deduce de los datos aportados en el texto, y surge del filtrado de los datos iniciales tras seleccionar a los doscientos investigadores con más publicaciones, de modo que el porcentaje que ha quedado como conclusión se refiere a las personas que más publican de su universo.

La exposición de resultados en el artículo es sumamente confusa; en sus métodos indican que la selección realmente se estableció con 908 científicos que habían publicado al menos 20 trabajos (en su mayor parte contribuyentes a los informes del IPCC), de los que solamente 817 fueron identificados como conformes con las tesis del IPCC (su clave es $\mathrm{CE}$ ), de manera que el porcentaje de consenso de los "expertos" seleccionados sería de $89,9 \%$ (817/908), que sobre el total analizado de 1372 se reduce al 59,5\%, ascendiendo el porcentaje de no conformes al 39,5\%. Por ello su cifra de consenso redondeada a 96$97 \%$ no es clara en su origen, y se refiere a un reducido grupo del total de investigadores estudiado.

La cifra de consenso global que se suele señalar a este trabajo es del $66 \%$ (véase Cook et al., 2016; Tol et al., 2016, entre otros); esta cifra no aparece en todo el texto y parece ser el resultado de dividir la cantidad de 908 expertos seleccionados por haber publicado más de 20 artículos, entre el total encuestado de 1372, tal como hemos comentado previamente, pero recordemos que no todos los 908 estuvieron conformes, solo 817 y su porcentaje sobre el total sería ligeramente inferior: 59,5\% (Tabla 3).

Tabla 3. Porcentaje de consenso según Anderegg et al. (2010) y reanalizado en este trabajo.

\begin{tabular}{|l|c|c|c|}
\hline \multicolumn{1}{|c|}{ Número } & Total & Anderegg et al. & Reanalizado \\
\hline Original & 1372 & & $908 / 1372(66,1 \%)$ \\
\hline Filtrado & 908 & $817 / 908(89,9 \%)$ & \\
\hline Recalculado & 1372 & & $817 / 1372(59,5 \%)$ \\
\hline
\end{tabular}

Tres cartas al editor de la revista donde se publicó han criticado con dureza este texto demostrando que no pasó la revisión por pares. O’Neil (2010) lo ha sido especialmente con las formas en que el trabajo califica a los no conformes con el consenso. Aarstad et al. (2010) escribe que es una muestra del paradigma dominante y de su efecto sobre lo 
que se publica, de manera que un área de conocimiento puede quedar controlada por la aparente credibilidad, prominencia y ubicuidad de un grupo determinado de personas, lo que sin duda no es criterio de veracidad, en una variante del conocido "efecto San Mateo" (Merton, 1968). Estos autores no niegan que exista un consenso sobre los asuntos analizados, pero sí afirman que un paradigma dominante no tiene por qué ser cierto, y que además el consenso no ha sido demostrado. En términos parecidos incluso más enérgicamente se manifestó Bodestein (2010), quien no solo criticó el método de otorgar preeminencia y validez a la cantidad de publicaciones, sino que advirtió que eminentes climatólogos que disienten de alguna manera con las tesis defendidas no fueron considerados, y concluye con una dura crítica a la editorial de la revista PNAS por haber publicado dicho trabajo ante los defectos detectados. En Legates et al. (2013b) también se pueden encontrar críticas generales sobre el texto especialmente centradas en el concepto de consenso empleado.

En resumen, el trabajo presenta como conclusiones generales datos parciales que proceden de filtrados sobre el total (que posteriormente pasan casi inadvertidos), así como basados en la consideración del criterio de autoridad y cantidad como pruebas científicas. Resumidamente, lo que realmente demuestra el estudio es que aquellos autores más cercanos a las tesis del IPCC publican más. Por ello sus conclusiones pudieran haber sido escritas del modo siguiente: "817 climatólogos en su mayoría vinculados al IPCC y que han publicado más de 20 artículos expresan su acuerdo sobre...”.

\subsection{Quantifying the consensus on anthropogenic global warming in the scientific litera- ture (por Cook et al., 2013)}

El texto que sin duda ha captado más atención es el de Cook et al. (2013) con más de 500.000 descargas en internet y algunos centenares de citas en la base de datos ISI. El estudio se realizó mediante una revisión y posterior encuesta de 11.944 publicaciones (periodo 1991-2011) referidas a 29.083 investigadores y fue elaborado por los miembros de un blog denominado skeptical science. Los siete criterios de clasificación empleados básicamente se reducen a cuatro grupos: a favor, sin posición, en contra y otros. La cifra global de consenso que dan los autores es $97,1 \%$ y $98,4 \%$ respectivamente para el número de publicaciones y autores, pero este dato se refiere solamente a aquellos casos en que se expresó una opinión. Este hecho es muy importante.

Tal como se indica en el texto, en el 66,4\% de trabajos, y también en el 64,6\% de autores (pero este dato no aparece en el resumen), no se encontró un posicionamiento y fueron excluidos sin más del cálculo del porcentaje de consenso. Así, el valor que aparece como conclusión desde el resumen surge de comparar 3896 artículos sobre un total de 4014, y en el número de autores con respuesta favorable de 10188 respecto al total parcial de 10356 (Tabla 3, Cook et al., 2013). Pero si se computan tanto el número de textos como de autores con una opinión favorable sobre el total original del estudio (11944 artículos y 29286 autores), los porcentajes de consenso descienden al 32,6\% en publicaciones y al $34,8 \%$ en autores, valores que aparecen en la citada Tabla 3 del original y sobre los que los autores pasan prácticamente por encima sin comentar. 
En una segunda fase se seleccionaron 8547 autores a quienes se les envió un cuestionario y de quienes se recibieron 1200 respuestas, finalmente filtradas en 2142 trabajos y 1189 autores, en los que encontraron un consenso de 97,2\% y 96,4\% (respectivamente en trabajos y autores). Los porcentajes de nuevo fueron calculados tras eliminar del total aquellos que no expresaron una posición concreta sobre las preguntas realizadas, es decir en el primer caso el porcentaje se calculó entre 1342 respuestas favorables sobre la suma de 1381 artículos, y entre 746 autores sobre un total de 774 con opinión manifiesta sobre la preguntas, y no sobre el conjunto de la sub muestra ya filtrada de 2142 trabajos y 1189 autores (Tabla 4, Cook et al., 2013). Sin embargo en el propio texto se calculan los porcentajes sobre el total del filtrado que proporcionan valores de consenso en torno al $62 \%$ tanto en trabajos como autores, siendo un $30 \%$ el porcentaje que no manifiesta posición favorable o contraria.

Tabla 4. Porcentajes de consenso en Cook et al., 2013, y re-análisis.

\begin{tabular}{|l|c|c|c|c|c|c|}
\hline & \multicolumn{3}{|c|}{ Abstract } & \multicolumn{3}{c|}{ Autores } \\
\hline 1 Pregunta & $\mathbf{N}^{\mathbf{0}}$ & $\%$ & $\begin{array}{c}\% \\
\text { sugerido }\end{array}$ & $\mathbf{N}^{\mathbf{0}}$ & $\%$ & $\begin{array}{c}\% \\
\text { sugerido }\end{array}$ \\
\hline A favor & 3896 & 32,6 & $97,1 \%$ & 10188 & 34,8 & $98,4 \%$ \\
\hline Sin posición & 7930 & 66,4 & & 18930 & 64,6 & \\
\hline En Contra & 78 & 0,7 & & 124 & 0,4 & \\
\hline Inciertos & 40 & 0,3 & & 44 & 0,2 & \\
\hline & 11944 & & & $29286 *$ & & \\
\hline 2 Pregunta & & & & & & \\
\hline A favor & 1342 & $62,7 \%$ & $97,2 \%$ & 746 & $62,7 \%$ & $96,4 \%$ \\
\hline Sin posición & 761 & $35,5 \%$ & & 415 & $34,9 \%$ & \\
\hline En Contra & 39 & $1,8 \%$ & $2,8 \%$ & 28 & $2,4 \%$ & $3,6 \%$ \\
\hline & 2142 & & & 1189 & & \\
\hline Reanalizados & & & & & & \\
\hline Pregunta 2 & $1342 / 11944$ & & $11,2 \%$ & $746 / 29286$ & & $2,6 \%$ \\
\hline
\end{tabular}

* La cifra total de autores de 29083 que aparece en el texto de Cook et al. (2013) es incorrecta a partir de sus parciales, vide Tabla 3. Legates (2013b) la muestran desglosada por categorías. Los datos mostrados en las filas de las dos preguntas señaladas son los originales en Cook et al. (2013).

Por último, si se comparan los datos del filtrado con los totales originales los porcentajes de consenso descienden a valores minúsculos del 11,2\% en el caso de trabajos publicados (1342/11.944) y a 2,6\% en el caso de autores (746/29.286), que es la opinión manifestada por Bast et al. (2013) y concuerda con el método que Cook et al. (2016) aplican para estimar los valores globales de consenso tras filtrar los datos originales al trabajo de Anderegg et al. (2010) ya citado, aunque en un texto posterior defienden su método ante las críticas recibidas por Tol (2014 a y b). Notablemente además la cifra del total de autores incluida en el texto no concuerda con la que se deduce de la Tabla 3 cuya 
suma es de 29.286 (y no ha sido advertida ni corregida hasta el momento, pero aparece correctamente en Legates, 2013b).

En resumen, el consenso que propone el trabajo de Cook et al. (2013) surge de una opinión que inicialmente descarta a más del $60 \%$ de la población analizada (Tabla 1) en su primera parte, a un tercio en la segunda, y en caso de considerar aceptable el filtrado que realizan, su consenso sería el de 746 personas.

Las críticas que se han vertido contra este texto demuestran que es un caso extremo de justificación de una conclusión preconcebida, y los argumentos esgrimidos por sus autores (Cook et al., 2014, 2015) ante las críticas recibidas por Tol (2014a y b; 2016), Dean (2015) y Legates et al. (2013a y b), así lo indican.

Tol (2014a y b, 2016) ha entrado más en detalle sobre los métodos y la calidad de los datos que en otros aspectos, insistiendo en (i) la omisión de no señalar con claridad en las conclusiones que el $67 \%$ de los encuestados no postulaban consenso alguno, (ii) que la acumulación de trabajos o autores sobre un particular no son prueba de lo que se quiere demostrar, y (iii) que la mayoría de los textos analizados realmente no tratan de las causas sino de estudios sobre los efectos y medidas ante eventuales cambios; este autor insiste que no duda que pueda existir dicho consenso, sino que este trabajo no lo demuestra. Las últimas publicaciones de todos ellos parecen indicar que su debate particular aún no ha terminado. Por su parte Dean (2015) ha criticado el proceso de clasificación en su origen, y Legates et al. (2013a y b), tras revisar los listados del original estimaron un paupérrimo $0,34 \%$ de consenso en el conjunto de publicaciones, señalando que es ilícito descartar a aquellos que no manifiestan una opinión como favorables del cómputo global. También estos autores han criticado el proceso de clasificación y la ausencia de información sobre los clasificadores. Este hecho es de especial importancia dado que una defensa habitual de los firmantes es que su objetivo ha sido buscar el consenso manifestado por los "expertos, pero los "revisores de textos" proceden de un blog caracterizado por su virulencia y ataque a cualquier opinión contraria a las tesis del IPCC, grupo del que nunca se ha demostrado su cualificación en climatología.

Por todo ello, sus conclusiones podrían haber sido escritas del modo siguiente "tras descartar a más del sesenta por ciento de la muestra analizada, 746 expertos en clima afirman ...".

\subsection{Scientists' views about attribution of global warming (por Verheggen et al., 2014)}

Verheggen et al. (2014) analizaron 1868 investigadores de un total inicial de 7555 con publicaciones en el periodo 1991-2011. En la encuesta entre otras cuestiones se analizaron dos preguntas clave: si más de la mitad del aumento térmico de la segunda mitad del siglo XX estaba causado por las emisiones humanas, y qué grado de fiabilidad se tenía sobre esta afirmación. En el resumen inicial indicaron que habían encontrado un consenso de más del $90 \%$ entre los expertos en climatología que habían publicado más de 10 artículos sobre ambas cuestiones, pero los valores de consenso globales aparecen en mitad del texto con cifras de 65,9\% y 89,2\% respectivamente para las preguntas 1 y 2 . 
En general el trabajo se pierde en una sucesión de porcentajes sobre datos filtrados del total original, con una exposición poco clara y muy difícil de seguir en sus argumentaciones, entre otras cosas porque no aparece una sola tabla de resultados que de al lector una idea de la magnitud a que se refiere cada porcentaje, y porque los totales, cuando se muestran, aparecen en las leyendas de las figuras con sus diferentes subgrupos pero la suma de los parciales no coincide con el total (Figura 1, 2, 4, etc). Tomando los datos que se ofrecen en el texto, los valores de consenso global favorables a la segunda pregunta serían 910 expertos (según la Figura 4) como suma de dos grupos que, respecto al total filtrado de 1222 , no produce el $84 \%$ señalado en el texto sino un 74,4\% (Tabla 5).

Tabla 5. Estimación del consenso en Verheggen et al., 2014 (manuscrito), Strengers et al., 2015 (informe), y re-análisis.

\begin{tabular}{|c|c|c|c|c|c|c|}
\hline & \multirow{2}{*}{ Total } & \multicolumn{3}{|c|}{ Efecto humano } & \multicolumn{2}{|c|}{ Confianza } \\
\hline & & $>50 \%$ & $<50 \%$ & Otros & Alta & Baja \\
\hline Original & 1868 & $\frac{1231}{(65,9 \%)}$ & $\begin{array}{c}220 \\
(11,8 \%)\end{array}$ & $\begin{array}{c}418 \\
(22,3 \%)\end{array}$ & & \\
\hline $\begin{array}{l}\text { Filtrado } \\
\text { (manuscrito) }\end{array}$ & $\underline{1222}$ & & & & $\begin{array}{c}910 \\
(74,4 \%)\end{array}$ & \\
\hline & $\underline{1868}$ & & & & $\begin{array}{c}910 \\
(48,7 \%) \\
\end{array}$ & \\
\hline Filtrado (informe) & $\underline{1222}$ & \multirow{2}{*}{\multicolumn{3}{|c|}{$\begin{array}{l}\text { Respuestas afirmativas a ambas } \\
\text { preguntas (1091) }\end{array}$}} & $\begin{array}{l}1091 \\
(89.2 \%) \\
\end{array}$ & $\frac{131}{(10,8 \%)}$ \\
\hline Recalculado & 1868 & & & & $\begin{array}{c}1091 / 1868 \\
(58,4 \%)\end{array}$ & \\
\hline
\end{tabular}

El trabajo, sin embargo, puede reconstruirse mediante el informe de la investigación original publicado poco después por los mismos autores (Strengers et al., 2015), y su análisis permite verificar que en la publicación se ha producido un baile de cifras respecto a los datos en la segunda pregunta no corregido. Como acabamos de comentar, en la Figura 4 del texto original el total de respuestas de 1222 se refiere a aquellos encuestados que expresaron que al menos la mitad del aumento de las temperaturas tenía origen en los gases de efecto invernadero. De este subgrupo los que están conformes con una elevada fiabilidad del efecto humano sobre las temperaturas suman 910 personas, mientras los no conformes serían 312. En la citada Figura 4 el total que se indica del total de respuestas conformes de 1222 no se corresponde con la suma de los parciales que es de 1127, pero sí los 910 conformes. En consecuencia, la conformidad ante la pregunta inicial de que el efecto humano sobre el aumento de las temperaturas supone al menos la mitad del observado (conservando el total de 1222) llevaría a un consenso ya comentado de $74,4 \%$ (910/1222), que si se considerase sobre el total de respuestas se reduciría al $48,7 \%(910 / 1868)$.

El problema es que las 910 respuestas a la pregunta 2 no aparecen en el informe, que pasan a ser 1091, y que al relacionarse con su total filtrado de 1222 producen el 89,2\% de consenso indicado, que tampoco se corresponde con el total general a la segunda pregunta del artículo. Advertimos que en caso de mantenerse estas cifras, sobre el total de 1868 el consenso se reduciría a $58,4 \%$. 
La razón de estas discrepancias y bailes de datos estriba en que el informe presenta dos grupos de contestaciones en la segunda pregunta. El primero con 1222 respuestas y el segundo con 217, y en el artículo solamente se presentan los primeros, pero parecen calculados con los dos grupos de datos.

Este juego de porcentajes ha sido criticado por Idso et al. (2015) señalando que el valor final estaría en torno a un $40 \%$ de consenso sobre los datos originales. Otras críticas fueron destacadas por Duarte (2014), especialmente las relacionadas con el método de selección que provocó que numerosos trabajos irrelevantes se incluyeran pese a no tratar los objetivos del estudio y ser realizados por personas ajenas a la climatología.

En resumen, el texto además de ser muy confuso en su exposición y con incoherencias con el informe original, aplica una vez más sucesivos filtrados que elevan a conclusiones globales resultados parciales, lo que supone descartar una parte de la muestra estudiada. Por otro lado las discrepancias entre el texto y el informe del que surge sugieren errores y descuidos de los autores, así como un escaso celo de la revista que lo publicó. Sus conclusiones podrían haberse escrito en términos como "algo más de la mitad de 1868 científicos consultados concuerdan en..." (con datos del informe), y según lo publicado "algo menos de la mitad de 1868 científicos consultados concuerdan en...", o simplemente “.. 910 científicos están conformes con..."

\subsection{Otros textos}

Los textos que hemos comentado en los apartados previos son sin duda los más citados, si bien existen otros trabajos en los que se ha estimado el valor de consenso.

Rosemberg et al. (2010) analizaron las publicaciones de 13 revistas del periodo 1995-2004 buscando entre sus autores climatólogos de EEUU (986) a los que se envió una encuesta que contestaron 468. Las respuestas recibidas indicaron que un $61,6 \%$ (270 personas) estaba muy conforme (strong agree) con la existencia de un aumento de las temperaturas, y el 32,4\% (142) manifestaron su conformidad (agree). A la pregunta de si las actividades humanas podían acelerar dicho aumento el 49,1\% (213 personas) manifestó mucha conformidad y el 39,3 (170 personas) conformidad. Pero a partir de estas conclusiones equipara estos resultados con los postulados del IPCC, a saber: (1) el calentamiento del sistema climático es inequívoco, y (2) el aumento de las temperaturas medias es muy probable (>90\%) que se deba a las emisiones humanas de gases invernadero, que no fueron las preguntas realizadas, especialmente la segunda, y es llamativo que el grupo clasificado simplemente con un "conforme" aumentara en la segunda pregunta respecto a la primera. También en el trabajo se identifican lo que definen como temas en desacuerdo, que concretamente se resumen en las tasas propuestas del aumento térmico y las variaciones espaciales, aunque curiosamente no dan cifras sobre estos asuntos y en la Figura 3 el porcentaje que estima que el cambio del clima es un problema moderadamente significativo es de un $30 \%$ aproximadamente.

Lefsrud y Meyer (2012) analizaron 1077 respuestas de profesionales de la ingeniería y geociencias de sociedades de EEUU y Canadá, y encontraron que el 
$99,4 \%$ estaba conforme en afirmar que las temperaturas habían aumentado, pero esta respuesta no supone consenso alguno en la línea que estamos comentando; otros datos aportados por estos autores indicaron que un 44,4\% consideraba que el efecto humano sobre el clima era pequeño, no dominante (que sí estaría más cerca de los planteamientos citados), y un $36 \%$ consideraba que el debate sobre los efectos humanos en el clima no estaba cerrado, cifras estas lejos del consenso auspiciado por los trabajos previos. En el texto los autores explícitamente aceptan la ausencia de consenso al escribir “... en realidad el consenso no es tal y hay mucha gente escéptica en un porcentaje no asumido, que procede de las más variadas profesiones" (traducción libre). Legates et al. (2013b) han sugerido la mayor calidad metodológica de este texto, si bien critican el argumento de fundamentarse en la opinión de expertos. Por otro lado el consenso sobre que las temperaturas han aumentado, que es la pregunta clave del análisis, es obvia en su respuesta.

Bray y von Storch (2013, y en sus informes de años previos) han constatado una variación temporal del llamado consenso y en general sus resultados no ofrecen cifras que permitan afirmar un consenso como el citado en los textos previos. Por descontado en sus análisis encuentran respuestas mayoritariamente afirmativas a que se ha producido un aumento reciente de temperaturas, y también a que el hombre pueda haber contribuido a dicho aumento térmico. En el informe de 2013 encuestaron a 283 personas expertas en climatología, y contabilizando las puntuaciones por encima o debajo del valor neutro 4 de su escala según la pregunta que sea, pueden destacarse por ejemplo (i) que el $60,5 \%$ de los encuestados no considera criterio relevante para valorar una investigación el número de publicaciones de un científico (pregunta 65), (ii) que más del $62 \%$ considera que el cambio del clima es tanto científico como político (pregunta 72), (iii) que más del $67 \%$ opina que el discurso relacionado con el mismo está dirigido por los sentimientos del público y los políticos más que por los científicos (pregunta 73), y (iv) que en realidad para el 52,5\% de encuestados la tarea de mayor importancia sería definir los problemas y las causas (atribución) del cambio del clima (pregunta 78). Respecto a lo que podríamos calificar de consenso (pregunta 27) el $80,8 \%$ declara en su escala de puntuación (1-7) un valor entre 5 y 7 a favor de que el cambio del clima sea de origen humano, valor que fluctúa respecto a informes de estos autores de años previos: así en 1996 fue del 40\% (539 personas), en 2003 del $50 \%$ (530 personas), y en 2008 del $83,5 \%$ (370 personas).

Stenhouse et al. (2014) publicaron una entrevista realizada a 1821 miembros de la American Meteorological Society (AMS) y calcularon un consenso del 52\%, que Bast (2013) ha corregido al 39\% a partir de una investigación previa de Maibach et al. (2012) en la que el $70 \%$ de los miembros de la citada asociación expresaban dudas sobre la gravedad del aumento térmico. En las tablas que proporciona el texto se advierte por ejemplo que en la selección de "climatólogos" el porcentaje expresado de consenso sobre el efecto humano (que es del 78\%) se debe a una población de 124 personas (aunque Cook et al., 2016 elevan el porcentaje a 93\%), que en el caso de meteorólogos el consenso (61\%) se debe a la opinión de 61 personas, y que en el total de climatólogos y meteorólogos (1203) el consenso es del 45,5\%. Si bien los datos del informe de 2012 no disienten prácticamente de 
los indicados por la corrección de Bast, su crítica debería matizarse por referirse a totales diferentes, aunque las encuestas se realizaron en el mismo universo (AMS), con poblaciones muy semejantes en sus totales y en periodos muy cercanos. Curiosamente Cook et al. (2016) en una de sus respuestas a Tol se refieren a este trabajo citando una cifra de consenso global en torno al $75 \%$ que se refiere de nuevo a totales parciales del estudio. Respecto al informe de Maibach et al. (2012), algunas de las respuestas sorprenden: el $89 \%$ opinó que las temperaturas habían aumentado en los últimos 150 años (pregunta 1) con un grado de certeza del 74\%; el 59\% opinó que el hombre era el principal causante, y el 11\% opinó que era una combinación de factores humanos y naturales (pregunta 3). Otras respuestas se relacionaron con el grado de preocupación (pregunta 6) que incluye un $30 \%$ muy preocupado y el $42 \%$ algo preocupado.

Cifras semejantes aparecen en el informe Pew Research Center elaborado para la American Association for Advancement of Science (Lainier, 2015), con un 87\% de acuerdo en el efecto humano sobre el aumento térmico, y un $77 \%$ de preocupación, a partir de una muestra de 3748 personas entrevistadas, si bien en el grupo de "expertos" el consenso del $93 \%$ surge de nuevo de un reducido número de personas (132).

Powel (2014) ha analizado 17.352 resúmenes del periodo 2012-2013 de 69.406 autores, y ofrece un dato sobre el consenso del 99,9\% sin especificar en el texto los métodos, los criterios de análisis y selección, y sin aportar dato alguno, de modo que la conclusión es poco creíble per se porque en su indagación el criterio de búsqueda es encontrar textos que indiquen que el $\mathrm{CO}_{2}$ no produce un efecto invernadero, lo cual raya lo absurdo a partir de un conocimiento básico y elemental de la química y climatología. Curiosamente este autor realiza una crítica demoledora del trabajo de Cook et al. (2013), tanto al método empleado, que califica de poco fiable, como por la ausencia de una definición de lo que entiende por consenso. Esta ausencia de concreción de lo que se entiende por consenso es generalizada entre todos los autores, excepto en el caso de Powel, para el cual consenso es sinónimo de unanimidad.

Carlton et al. (2015) encuestaron a 1865 investigadores obteniendo 698 respuestas. Un 93,6\% estuvo conforme en asegurar que la temperatura había aumentado, y de ellos 641 personas $(98,2 \%)$ indicó que la responsabilidad del hombre era evidente, ofreciendo un valor global de $91,9 \%$ de acuerdo. De nuevo en sus numerosas tablas se producen filtros si bien claramente especificados, de modo que sus resultados deben leerse con estas precauciones. Pero estos datos tampoco suponen una certificación del consenso porque no se especifica el grado de intervención ni de qué modo se efectúa, es decir de nuevo nos encontramos con una pregunta obvia y abierta que no presupone grado de concreción alguno. La inspección detallada del texto además demuestra que la mayoría de personas encuestadas procedían del campo de la biología (28\%) y que los relacionados con la climatología y meteorología apenas llegaban al $3 \%$. Esta circunstancia se observa con claridad en el texto de Tol (2016) que desglosa los valores absolutos de respuestas y porcentajes de los diferentes grupos de categorías profesionales. En suma, el trabajo presenta un consenso de que el hombre contribuye 
al cambio del clima ( el $\mathrm{CO}_{2}$ atmosférico efectivamente absorbe energía de onda larga y re-emite en todas direcciones. Como ocurre en otros trabajos publicados sobre el particular, el mayor problema es que los expertos encuestados proceden en su mayoría de investigadores de impactos y de estrategias de mitigación ante un cambio del clima, es decir no estudiosos de la naturaleza y razones de dicho cambio. En suma el trabajo tampoco es concluyente sobre lo que pretendía demostrar. El texto demuestra además el grado de ideologización que ha alcanzado el debate, puesto que trata de discriminar la opinión sobre el consenso con una clara intención de demonizar determinadas opciones políticas. Esta línea argumental ya la expresaron Kahan et al. (2011) al señalar cifras de consenso (entre el $45 \%$ y $15 \%$ ) sobre la responsabilidad humana en el aumento de las temperaturas que relacionaron con atribuciones ideológicas y culturales. En la misma línea se encuentran también los textos de Bedford (2010), Bedford y Cook (2013), Benestad et al. (2013), Kahan (2015) y Lewandowsky et al. (2013, 2016), muestras del evidente grado de ideologización que el debate ha alcanzado.

Liu et al. (2015) analizaron 1350 testimonios de 253 audiencias en el Congreso de EEUU (periodo 1969-2007) de los que 752 (55,7\%) fueron de científicos, y catalogaron su opinión sobre si existe un cambio del clima y qué efecto tiene el hombre, pero sin señalar causa concreta. Los resultados globales del estudio indicaron que un $44,3 \%$ de todos los testimonios estaban a favor de la primera pregunta, y un $24,7 \%$ de la segunda; sin embargo en el resumen aparecen las cifras de $85,1 \%$ y $78,4 \%$ como los porcentajes de respuestas a las dos preguntas dadas por los expertos, porcentaje que se han vuelto a calcular sobre un total parcial descontando todas aquellas respuestas que en las dos preguntas no habían manifestado una opinión, y que suman 647 y 925 personas en cada caso (véase Tablas 1 y 2 del original). Así, al contabilizar las opiniones de los científicos, de un total de 752 expertos opinaron favorablemente a las dos preguntas 384 y 220 . Estas cifras sobre el total de 752 suponen respectivamente $51,1 \%$ y $29,3 \%$ de respuestas afirmativas a ambas preguntas, pero de nuevo se recalcularon descontando el total de respuestas sin posicionamiento (306 y 471), es decir las cifras que se ofrecen como consenso proceden de un filtrado de los datos, que incluso en el caso de los considerados científicos descarta a más de un $40 \%$ en la primera pregunta y a más del $60 \%$ en la segunda, de modo que los porcentajes tras este descuento llegan a las cifras indicadas de $86,1 \%$ y $78,3 \%$. De nuevo si se contabiliza el porcentaje de las respuestas positivas de los científicos sobre el total analizado (1350) las cifras bajan al $28,4 \%$ y $16,3 \%$ respectivamente ante ambas preguntas

Finalmente, Losch (2016) ha observado que el porcentaje de consenso en la opinión pública, desde la publicación de Powel, decrece argumentando que lo que hace falta es ser más convincente en la exposición.

La Tabla 6 resume los principales resultados de los trabajos que se han comentado, los porcentajes "globales de consenso" sugeridos, los indicados por los autores que los han criticado y los recalculados en el presente texto cuando se ha podido a partir de los datos originales. 
Tabla 6. Valores porcentuales de consenso originales en diferentes autores y reanalizados.

\begin{tabular}{|c|c|c|c|c|c|}
\hline & Método & Observaciones & $\begin{array}{c}\% \\
\text { Asumido } \\
\end{array}$ & Cálculos & $\begin{array}{c}\% \\
\text { Estimado }\end{array}$ \\
\hline \multirow{2}{*}{$\begin{array}{l}\text { Oreskes, } \\
2004\end{array}$} & \multirow{2}{*}{928 abstract } & \multirow{2}{*}{6 grupos } & $75 \%$ & $\begin{array}{l}\text { - Peiser, } 2005 \text { (igual periodo y } \\
\text { método), } 1117 \text { abstract, } 322 \\
\text { coincidentes con categorías 1-2 } \\
\text { Oreskes. }\end{array}$ & $29,9 \%$ \\
\hline & & & & $\begin{array}{l}\text { - Schulte, } 2008 \text { (periodo } 2004- \\
\text { 2008), } 539 \text { abstract, } 282 \text { en } \\
\text { categorías 1-2 Oreskes }\end{array}$ & $52,3 \%$ \\
\hline \multirow{4}{*}{$\begin{array}{l}\text { Doran y } \\
\text { Zimmerman, } \\
2009\end{array}$} & \multirow{4}{*}{$\begin{array}{c}3146 \\
\text { encuestas }\end{array}$} & \multirow{2}{*}{$\begin{array}{c}\text { Aumento } \\
\text { Temperatura }\end{array}$} & $96,2 \%$ & $\begin{array}{l}\text { - Calculado con } 76 \text { climatólogos, } \\
\text { sobre total de } 79 \text {. }\end{array}$ & \\
\hline & & & & $\begin{array}{l}\text { - Calculado con } 76 \text { climatólogos } \\
\text { sobre total de } 3146\end{array}$ & $2,4 \%$ \\
\hline & & \multirow{2}{*}{$\begin{array}{l}\text { Impronta } \\
\text { humana }\end{array}$} & $97,4 \%$ & $\begin{array}{l}\text { - Calculado con } 75 \text { climatólogos } \\
\text { sobre total de } 77\end{array}$ & \\
\hline & & & & $\begin{array}{l}\text { - Calculado con } 75 \text { climatólogos } \\
\text { sobre total de } 3146\end{array}$ & $2,3 \%$ \\
\hline \multirow{3}{*}{$\begin{array}{l}\text { Anderegg et } \\
\text { al. } 2010\end{array}$} & $\begin{array}{c}1372 \\
\text { encuestas }\end{array}$ & \multirow{3}{*}{$\begin{array}{l}\text { Conformidad } \\
\text { con tesis IPCC }\end{array}$} & $89,9 \%$ & $\begin{array}{l}\text { - Calculado como } 817 \text { de } 908 \\
\text { expertos conformes }\end{array}$ & \\
\hline & \multirow{2}{*}{$\begin{array}{l}\text { Filtrados } \\
\quad 908\end{array}$} & & $66,1 \%$ & $\begin{array}{l}\text { - Calculado como } 908 \text { expertos } \\
\text { sobre } 1372\end{array}$ & \\
\hline & & & & $\begin{array}{l}\text { - Calculado como } 817 \text { de } 1372 \\
\text { expertos conformes }\end{array}$ & $59,5 \%$ \\
\hline \multirow{5}{*}{$\begin{array}{l}\text { Cook et al., } \\
2013\end{array}$} & \multirow{2}{*}{$\begin{array}{l}28286 \\
\text { autores }\end{array}$} & & $98,4 \%$ & $\begin{array}{l}\text { - Calculado como } 10.188 \text { expertos } \\
\text { favorables, sin contabilizar "el } \\
\text { grupo sin opinión" }\end{array}$ & \\
\hline & & & & $\begin{array}{l}\text { - Calculado como } 10.188 \text { expertos } \\
\text { sobre total de muestra de } 28.286\end{array}$ & $34,8 \%$ \\
\hline & \multirow{3}{*}{$\begin{array}{l}\text { Filtrados } \\
1189\end{array}$} & & $96,4 \%$ & $\begin{array}{l}\text { - Calculado como } 746 \text { favorables } \\
\text { sobre } 774 \text { que opinan }\end{array}$ & \\
\hline & & & & $\begin{array}{l}\text { - Calculado como } 746 \text { favorables } \\
\text { sobre selección } 1189\end{array}$ & $62,7 \%$ \\
\hline & & & & $\begin{array}{l}\text { - Calculado como } 746 \text { favorables } \\
\text { sobre total } 28.286 \\
\end{array}$ & $2,6 \%$ \\
\hline \multirow{5}{*}{$\begin{array}{l}\text { Verheggen } \text { et } \\
\text { al., } 2014 \mathrm{y} \\
\text { Strengers } \text { et } \\
\text { al., } 2015\end{array}$} & $\begin{array}{c}1868 \\
\text { expertos }\end{array}$ & $\begin{array}{c}\text { La mitad del } \\
\text { aumento térmico } \\
\text { tiene origen } \\
\text { humano } \\
\end{array}$ & $65,9 \%$ & $\begin{array}{l}\text { - Calculado con } 1231 \text { expertos } \\
\text { favorables sobre total } 1868\end{array}$ & \\
\hline & \multirow{4}{*}{$\begin{array}{l}1222 \\
\text { filtrados }\end{array}$} & \multirow{4}{*}{$\begin{array}{l}\text { Confianza en la } \\
\text { pregunta previa }\end{array}$} & $89,2 \%$ & $\begin{array}{l}\text { - Calculado como } 1091 \\
\text { expertos favorables sobre total } \\
\text { seleccionado } 1222 \text { (informe) }\end{array}$ & \\
\hline & & & & $\begin{array}{l}\text { - Calculado como } 1091 \text { expertos } \\
\text { sobre total } 1868 \text { (informe) }\end{array}$ & $58,4 \%$ \\
\hline & & & & $\begin{array}{l}\text { - Calculado como } 910 \text { expertos } \\
\text { sobre total seleccionado de } 1222 \\
\text { (publicación) }\end{array}$ & $74,4 \%$ \\
\hline & & & & $\begin{array}{l}\text { - Calculado como } 910 \text { expertos } \\
\text { sobre total de } 1868\end{array}$ & $48,7 \%$ \\
\hline \multirow{4}{*}{$\begin{array}{l}\text { Rosember et } \\
\text { al., } 2010\end{array}$} & \multirow{4}{*}{468 autores } & \multirow{2}{*}{$\begin{array}{c}\text { Aumento } \\
\text { Temperaturas }\end{array}$} & $94,0 \%$ & - 412 personas sobre 438 respuestas & \\
\hline & & & & $\begin{array}{l}\text { - } 412 \text { personas sobre } 468 \text { total } \\
\text { respuestas }\end{array}$ & $88,0 \%$ \\
\hline & & \multirow{2}{*}{ Efecto humano } & $88,5 \%$ & - 383 sobre 433 respuestas & \\
\hline & & & & - 383 sobre 468 total respuestas & $81,8 \%$ \\
\hline
\end{tabular}




\begin{tabular}{|c|c|c|c|c|c|}
\hline & Método & Observaciones & $\begin{array}{c}\% \\
\text { Asumido } \\
\end{array}$ & Cálculos & $\begin{array}{c}\% \\
\text { Estimado }\end{array}$ \\
\hline $\begin{array}{l}\text { Stenhouse } \text { et } \\
a l, 2014\end{array}$ & $\begin{array}{c}1821 \\
\text { encuestas }\end{array}$ & $\begin{array}{c}\text { Aumento } \\
\text { Temperaturas }\end{array}$ & $52,0 \%$ & - Corregido por Bast (2013) & $39,0 \%$ \\
\hline \multirow{10}{*}{$\begin{array}{l}\text { Liu et al., } \\
2015\end{array}$} & \multirow{10}{*}{$\begin{array}{c}1350 \\
\text { testimonios }\end{array}$} & \multirow{5}{*}{$\begin{array}{l}\text { Aumento } \\
\text { Temperaturas }\end{array}$} & $44,3 \%$ & $\begin{array}{l}\text { - Calculado sobre total de } \\
\text { respuestas }(598 / 1350)\end{array}$ & \\
\hline & & & $85,1 \%$ & $\begin{array}{l}\text { - Calculado sobre respuestas con } \\
\text { opinión }(598 / 703)\end{array}$ & \\
\hline & & & $86,1 \%$ & $\begin{array}{l}\text { - Calculado según respuestas con } \\
\text { opinión de científicos }(384 / 446)\end{array}$ & \\
\hline & & & $51,1 \%$ & $\begin{array}{l}\text { - Calculado sobre total de } \\
\text { respuestas de científicos } \\
(384 / 752)\end{array}$ & \\
\hline & & & & $\begin{array}{l}\text { - Calculado sobre total de } \\
\text { respuestas de científicos y total } \\
\text { del estudio }(384 / 1350)\end{array}$ & $28,4 \%$ \\
\hline & & \multirow{5}{*}{ Efecto humano } & $24,7 \%$ & $\begin{array}{l}\text { - Calculado sobre total de } \\
\text { respuestas }(333 / 1350)\end{array}$ & \\
\hline & & & $78,4 \%$ & $\begin{array}{l}\text { - Calculado sobre respuestas con } \\
\text { opinión }(333 / 425)\end{array}$ & \\
\hline & & & $78,3 \%$ & $\begin{array}{l}\text { - Calculado según respuestas con } \\
\text { opinión de científicos }(220 / 281)\end{array}$ & \\
\hline & & & $29,3 \%$ & $\begin{array}{l}\text { - Calculado sobre total de } \\
\text { respuestas de científicos } \\
(220 / 752)\end{array}$ & \\
\hline & & & & $\begin{array}{l}\text { - Calculado sobre total de } \\
\text { respuestas de científicos y total } \\
\text { del estudio }(220 / 1350)\end{array}$ & $16,3 \%$ \\
\hline \multirow{3}{*}{$\begin{array}{l}\text { Lefsrud y } \\
\text { Meyer, } 2013\end{array}$} & \multirow{3}{*}{$\begin{array}{c}1077 \\
\text { encuestas }\end{array}$} & $\begin{array}{c}\text { Aumento } \\
\text { Temperaturas }\end{array}$ & $99,4 \%$ & - No aportan información & \\
\hline & & Efecto humano & $63,6 \%$ & - No aportan información & \\
\hline & & Consenso NO & $36,0 \%$ & - No aportan información & \\
\hline
\end{tabular}

\section{Discusión}

\subsection{Consideraciones generales}

Los trabajos comentados, especialmente los cinco primeros analizados con más detalle por ser los más conocidos, tienen características comunes que han sido señaladas por las críticas de diferentes autores y que se pueden resumir en las siguientes:

La definición de consenso no es común, y por lo general los objetivos no están claramente fijados. Las preguntas de aquellos que realizan encuestas varían, lo que hace difícil su comparación, y en ocasiones son poco apropiadas para determinar cualquier consenso sobre la intervención humana en el clima por la emisión de gases. Es evidente que el hombre puede cambiar las condiciones del clima, pero no solamente por efecto de las emisiones sino también por el cambio de los usos del suelo entre otros modos; es evidente que ha habido un aumento térmico reciente, pero responder afirmativamente a esta pregunta o a la anterior no necesariamente implica una atribución predominante a las emisiones, y así sucesivamente. La falta de concreción ante los niveles de confianza que se preguntan es otra prueba de lo que decimos. 
Metodológicamente la revisión y clasificación de unos resúmenes es muy poco fiable como método para calificar la posición de los autores sobre cuestiones específicas. Es bien conocido que un resumen es una carta de presentación en la que se suelen "cargar las tintas" para atraer, primero, la atención de editor y revisores y, posteriormente, de potenciales lectores. Harlos et al. (2017) han señalado recientemente que los resúmenes exageran de alguna manera lo que el trabajo incluye, y que tras la presentación de los informes del IPCC se ha detectado un efecto de arrastre sobre determinados temas que aumentan su frecuencia; en suma un resumen no refleja con exactitud el tenor de los trabajos, menos la posición asignada a los autores ante las preguntas o clasificaciones propuestas, y existen modas sobre temas de investigación que pueden suponer un acúmulo de trabajos que de alguna manera sean reiterativos y quizá no viesen la luz con tanta facilidad en otro momento. Por otra parte son numerosos los autores que se han quejado por haber sido clasificados en determinadas categorías favorables al consenso a partir de los resúmenes de sus publicaciones, cuando el texto que se calificaba decía precisamente lo contrario.

Los criterios de clasificación de textos publicados en realidad esconden un argumento falaz. Los autores clasificados mayoritariamente estudian qué efectos podrían producirse (impactos) y qué medidas mitigadoras deberían realizarse dando por hecho los resultados obtenidos por los modelos, lo que constituye una clara falacia argumentum ad petitionem principii, y por ello no debería considerarse ni a sus autores como expertos climatólogos, porque su campo de trabajo no es las atribuciones del cambio del clima, ni sus estudios como prueba de consenso.

El argumento reiterado de que la mayoría crea la verdad, de que el consenso refleja la ciencia establecida, básica y fundamental (settled science, mainstream science), no es válido y remite a la falacia aristotélica argumentum ad populum. La ciencia por definición no puede ser ni es democrática; de la misma manera, sostener la validez del consenso por el argumento de que la opinión de los expertos fija la ciencia tampoco es válido y expresa otra falacia (argumentum ad verecundiam), sobre todo por el hecho de que los porcentajes de expertos se refieren muchas veces a personas seleccionadas cercanas a las tesis del propio IPCC. Sería lo mismo que preguntar a los seguidores de un equipo de fútbol si desean que gane la liga, simplemente no representan la población de aficionados a dicho deporte. El criterio de selección de expertos a partir del número de publicaciones tampoco resuelve el problema dada la redundancia que se ha podido observar en los índices h (Engqvist y Frommen, 2008) y a que tampoco el sistema de revisión de pares está exento de problemas (Allison et al., 2016; Lafambroise, 2016).

En aquellos casos en que se detectó una posición no declarada tanto en las publicaciones como en los autores, la norma habitual ha sido descartar este grupo en el cálculo de los porcentajes, lo que es un claro sesgo intencionado para contraponer a aquellos que declaran una posición a favor frente a los que se declaran contrarios. Tan válida es la posición a favor como contraria, y por supuesto la declaración de no tener seguridad ni en una u otra, que en muchos casos resulta ser la respuesta mayoritaria. En consecuencia, los porcentajes finales que llegan a emplearse como prueba del consenso global reflejan simplemente aspectos muy parciales de las investigaciones, dando lugar 
a que los valores sugeridos de consenso científico provengan de las opiniones de un reducidísimo número de personas, lo que contrasta con el anuncio de que los estudios son masivos. Cuando las críticas han incidido en estos aspectos la contestación ha sido invariablemente que se buscaba conocer la opinión de los expertos. Cook et al. (2016) se han esforzado en presentar porcentajes de atribución de climatólogos y meteorólogos, y las cifras que muestra indican que el consenso que obtienen procede de algunas centenas, y en ocasiones solamente unas decenas de personas. Lo mismo se puede observar en la revisión de Tol (2016), y en la Tabla 6 del presente texto.

En numerosos casos se detecta el intento de atribuir unas ideas políticas a las ideas enfrentadas, en una nueva falacia no descrita por Aristóteles pero que podríamos titular como argumentum per adscriptione; no se está en posesión de la verdad por ser más liberal o conservador, aunque no sean estas las categorías estrictamente analizadas, como tampoco el disentir de las tesis de la settled science implica que detrás esté un emporio petrolero.

Un fenómeno relevante que pasa en general desapercibido es que con el argumento de que la mayoría crea la verdad, y que esta verdad está sostenida por expertos (véase la crítica de Legates et al., 2013b), gran parte de los escritos sobre el consenso están redactados por personas ajenas a la climatología, que califican, ordenan, jerarquizan y evalúan el material que trabajan, pero que sistemáticamente aluden al juicio de expertos sobre el clima como criterio; su punto de partida es la settled science ya comentada, sobre la que no hay dudas porque está consensuada. Del mismo modo llama la atención el uso partidista de los argumentos según convenga, por ejemplo se utiliza en contra de los críticos que mucha de la información que manejan se publica fuera de los circuitos de las revistas con revisión de pares, aparece en blog de internet, y sus autores no pertenecen al ámbito académico o no son expertos (véase p.e. comentarios de Bedford, 2010, sobre algunos climatólogos), lo que no concuerda con sus propias filiaciones o con su crítica a la llamada literatura gris, si bien luego se apoya en numerosas ocasiones en ella (véase Lewandoswky et al., 2016).

Por último, llama la atención la facilidad con que estos trabajos han sido publicados en revistas tenidas por serias y con un riguroso sistema de revisión por pares ante las críticas recibidas que se han citado. Dados los defectos notados por los diferentes estudios críticos y señalados en el presente texto, cabe pensar en el interés de su publicación a toda costa. Todo ello supone un demérito al propio sistema de pares que en los propios textos comentados se alude sin embargo como uno de los modos de verificar la "ciencia correcta”.

\subsection{El consenso, el disenso y el debate}

El llamado cambio climático ha tenido uno de sus puntos más característicos en el denominado consenso que etimológicamente vendría a significar acuerdo en el paradigma expresado. Los textos objeto del presente ensayo han sido argumentados como prueba irrefutable de la tesis sostenida por el IPCC, en un argumento circular en el que los trabajos aluden al consenso manifestado por los textos del IPCC, y el IPCC 
argumenta sus resultados en el consenso de la aplastante mayoría de estudios (véase Bedford, 2010, entre otros muchos). Pero el debate no ha sido limpio y en esta situación se ha intentado acallar cualquier discrepancia por el medio que fuera; la historia del denominado "climagate" dejó ver algunas de las prácticas habituales, como evitar la publicación de textos contrarios a las tesis del IPCC, y son sobradamente conocidas con solo recordar la frase "... can't see either of these paper being in the next IPCC report... (we) will keep them out somehow, even if we have to redefine what the peer-review literature is!". Los defensores del planteamiento del IPCC, que en sus versiones más radicales reducen la hipótesis a que el hombre es el causante del cambio del clima por la emisión de $\mathrm{CO}_{2}$, llegan a lo absoluto al afirmar que se ha alcanzado la denominada "settled science", la ciencia definitiva, y que el debate está cerrado. En su argumentación afirman que (i) una cosa es disentir y otra crear infundios, (ii) que la discrepancia puede ser legítima e ilegítima, porque la discrepancia puede esconder oscuras intenciones ligadas a una conspiración para desinformar a la sociedad (Biddle y Leuschner, 2015), y por último (iii) se defiende la tesis argumentando que está basada en el consenso científico (Benestad et al., 2013; Bedford y Cook, 2013; Lewandowsky et al., 2013, 2016, entre otros). O dicho de otro modo: están en posesión de la absoluta verdad. Esta postura olvida que el propio informe IPCC de 2013 incluyó textos en los que indicaba una baja confianza de que hubieran aumentado el número de huracanes, las sequías, las lluvias torrenciales, y que el aumento del nivel marino no era el que se había indicado previamente.

El mayor descubrimiento de la ciencia ha sido informarnos de nuestra ignorancia (Harari, 2016). En ciencia recibe el nombre de hipótesis una aseveración fundamentada en el conocimiento existente, la lógica y la argumentación razonada, y también en el sentido común. Popper nos ha enseñado que ninguna hipótesis es cierta, y que solamente la mantenemos como válida (cursiva intencionada) en tanto que las pruebas a las que la sometemos no son capaces de rechazarla. Es decir, la duda y el escepticismo no es que sean sanos, son necesarios. Huxley dejo escrito: "The improver of natural knowledge absolutely refuses to acknowledge authority, as such. For him, skepticism is the highest of duties; blind faith the one unpardonable sin", y cuando Einstein fue advertido que se había escrito un libro firmado por cien científicos contra sus teorías (titulado "100 científicos contra Einstein”), contestó: “¿por qué cien?, con uno solo que tenga razón basta", idea similar a la expresada en otro contexto: "ningún dato prueba mis teorías, pero uno solo basta para rechazarlas"; Feymann también dejó sentenciado el valor de la duda al indicar que "cuando un dato, un experimento va contra la teoría, no importa quien la haya elaborado y sostenido, ni su autoridad, ni lo bonita que fuera, simplemente la teoría está mal, es incorrecta". En suma, una hipótesis es algo circunstancial, algo que si no es debatible pasa a ser un dogma que pertenece al campo de la teología, y la teología y la ciencia son cosas distintas pues, parafraseando a Galileo, "la primera nos enseña como ir al Cielo, y la segunda como se mueven los cielos".

En la ciencia además se consideran dos tipos de hipótesis, la llamada hipótesis nula y la hipótesis alternativa. La primera es aquella que resume el saber que está aceptado en un momento dado y es contra la que hay que "combatir" para que el conocimiento progrese; el avance podría ser a modo de revoluciones científicas como propuso Lakatos, 
los programas de Chandler, la falsación de Popper, o incluso mediante el "todo vale" de Feyerabend. Pero se suelen olvidar que lo que hay que tratar de combatir con alternativas es la hipótesis nula y que nuestras nuevas opciones, ideas o descubrimientos son la hipótesis alternativa que podemos defender solamente rebatiendo la hipótesis nula.

Ambos supuestos no se han observado en la corriente de opinión del llamado consenso sobre el cambio del clima que defiende las conclusiones de los informes del IPCC. Los informes IPCC, especialmente a partir del AR3 de 2001, han concluido afirmando con un grado de confianza muy elevado que el hombre ha cambiado el clima reciente por medio de las emisiones de combustibles fósiles, si bien existen numerosos estudios que ponen en duda especialmente dicho grado de confianza. Por su parte la corriente de opinión favorable al consenso de que las emisiones humanas son las responsables del aumento térmico y cambio del clima han partido de dichas conclusiones sin considerar que pudieran no estar definitivamente demostradas, violando el principio de que precisamente esa nueva hipótesis es lo que hay que demostrar de manera fehaciente por ser la hipótesis alternativa, y utilizando un principio incorrecto de demostración basado en que la mayoría de los científicos así lo afirman, olvidando que "la ciencia es un método de indagación de la verdad antes que una fuente de autoridad" (Lindzen, 2012).

Dado que numerosas de las predicciones sobre la evolución del clima no se han cumplido, no sólo es lícito sino absolutamente sensato y necesario plantearse dudas sobre dicha hipótesis, que recordemos basa sus fundamentos en un consenso y en el desarrollo de escenarios por medio de modelos. Diferentes hechos avalan entre otros muchos esta tesis; a modo de ejemplo solamente comentamos dos: el primero es la ausencia de aumento de las temperaturas en al menos los últimos 20-25 años en un periodo en que las emisiones han seguido creciendo de modo exponencial; el periodo comienza en torno a 1990 y ha sido denominado hiato, y ante esta anomalía se han elaborado decenas de argumentos para continuar defendiendo la hipótesis central, es decir argumentos ad hoc inaceptables en ciencia. El segundo es de mayor complejidad, y se relaciona con el efecto del $\mathrm{CO}_{2}$ sobre el forzamiento radiativo. Puesto que la baja troposfera está prácticamente saturada por la relación logarítmica del $\mathrm{CO}_{2}$ sobre la absorción de onda larga, todos los modelos predijeron un incremento del vapor de agua como consecuencia de dicho forzamiento que llevaría a un aumento térmico de la alta troposfera en baja latitud, hecho no confirmado por las medidas empíricas de satélites y globos sonda.

La reciente discusión sobre estos aspectos han llegado a posiciones difíciles de conciliar. Por un lado, los defensores del IPCC proponen que la hipótesis nula pase a ser que el aumento térmico es incontestablemente producto humano (Tremberth, 2011), y que su validez se sustenta en el consenso de la mayoría de los científicos. En el lado opuesto se ha planteado el abandono de las pruebas de hipótesis (Curry, 2011) ante las dudas existentes de que los cambios observados en el clima sean controlados por las emisiones humanas, y que en todo caso el criterio de una mayoría no asegura la verdad en la ciencia. Calificados como escépticos, deniers, o con palabras que rozan el insulto personal, este grupo aglutina a investigadores con tanto prestigio como otros incluidos 
en el primer grupo. Las dos posiciones han sido criticadas por Allen (2011), y Pielke et al., (2009) han ofrecido un planteamiento más holista.

La realidad es que el debate ha superado el ámbito académico y se ha establecido en todos los ámbitos de la sociedad opinando de cuestiones complejas, que ni siquiera los expertos reconocen entender completamente, desde organizaciones de todo tipo a actores políticos, no quedando ausentes las descalificaciones personales, los insultos y la presión por el medio que fuera sobre la opinión contraria (véase por ejemplo Lewandowsky et al., 2013, 2016, entre otros muchos).

\section{Recapitulación y conclusiones}

El análisis de los textos publicados sobre las cifras del consenso acerca del efecto que el hombre pueda tener sobre la evolución del clima por la emisión de gases, y especialmente su implicación en el aumento térmico detectado en la segunda mitad del siglo XX, demuestra que la cifra apabullante sostenida en torno al $97 \%$ es una simple reducción de los resultados y no refleja la realidad siquiera de las propias investigaciones. En general, todos los estudios presentan como resultados generales aspectos parciales de sus datos. Un somero análisis de los que aportan y el estudio de algunos textos que los han criticado sugieren que todos ellos presentan sesgos claros para demostrar una conclusión previa. Con los datos revisados, una estimación del $50 \%$ de consenso sobre el efecto del hombre en el cambio del clima parece ser más cercana a la realidad, si bien las discrepancias entre ellos son muy grandes.

Las opiniones favorables sobre el consenso, y sin duda las más virulentas, proceden en su mayoría de personas no cualificadas en el campo de la climatología o meteorología (es notable la abundancia de psicólogos y activistas de blogs en internet), las cuales evalúan y califican textos sobre el clima, que no dudan en defenderse de las críticas recibidas aludiendo a la baja o nula cualificación climatológica de quien les critica o expone una opinión contraria. Es interesante hacer notar que lo que pretendidamente se critica de las personas que sostienen opiniones contrarías, a saber: falta de cualificación y experiencia por una adscripción a institutos privados, o tener publicaciones no revisadas, no se aplica a los propios autores; esto es muy evidente en algunas de sus afiliaciones, así como en las variaciones de su afiliación en sucesivos trabajos, buscando cada vez más el marchamo de mundo académico.

Entre las características de los textos más conocidos sobre los que se pretende sostener el consenso de la hipótesis del IPCC sorprende sobre todo la falta de claridad en la exposición de los datos. De hecho es muy común no mostrar a qué cifra de personas o estudios se refieren los porcentajes, lo que impide hacerse una clara idea de la magnitud del consenso. Su examen detallado en ocasiones reduce a límites ridículos el consenso que se pretendía demostrar. Por ello podemos afirmar que el consenso sobre el origen del cambio del clima por efecto de las emisiones humanas no se demuestra en la serie de estudios que pretender probarlo, pues no son ni concluyentes en sus datos ni correctos en su realización, y en su inmensa mayoría presentan indicios claros de sesgos intencionados que imponen cautelas sobre los objetivos reales de los que los realizaron. 
Pero la crítica más determinante que se puede realizar es que pretender sostener una hipótesis científica por el efecto de la mayoría dominante no es un argumento correcto, pues ningún acuerdo o mayoría es prueba científica de la verdad, ya que esta nunca se encuentra tras una votación. La ciencia no puede avanzar acallando a quienes discrepan con el paradigma dominante, del que faltan por demostrar muchos de sus puntos clave, y menos llegar a las insinuaciones de connivencias con intereses ocultos, complot, e ilegitimidad del que discrepa, originando una situación en que la presión ejercida por determinados grupos es el factor que determina la verdad científica, y llevando a los no conformes a la prudencia del silencio ante una situación en la que una opinión contraria puede desencadenar ataques personales. Y tampoco es válida la justificación de la verdad basada en el criterio de algunos expertos.

Las temperaturas han aumentado durante el último siglo y las actividades humanas seguro que son capaces de modificar de alguna manera el sistema climático, pero el consenso no probará cómo. Quizá quien mejor expresó la situación actual ha sido Lovelock (2008) en dos frases que comparto: "Es ingenuo, científicamente hablando, pensar que podemos hacer predicciones relativamente precisas para el clima futuro, ... existen tantas incógnitas que está mal hacerlo", para más adelante concluir "Es casi seguro que no se puede lanzar un billón de toneladas de $\mathrm{CO}_{2}$ a la atmósfera sin que suceda algo desagradable".

Muy posiblemente el debate de los efectos de las emisiones humanas de $\mathrm{CO}_{2}$ en el clima lograría resultados óptimos si, en vez de realizarse en foros políticos o propagandísticos, se realizase de modo sereno en el ámbito de la ciencia, aceptando de antemano que siempre existirán legítimas discrepancias.

Mientras tanto, elaborar listas de científicos bajo el calificativo de deniers, y publicarlas en internet recuerda a los Libros Verdes de Aragón, las leyes alemanas de Nüremberg y a las listas del senador McCarthy no tan lejanas.

\section{Referencias}

Allen, M. 2011. In defence of the traditional null hypothesis: remarks on the Trenberth and Curry WIREs opinion articles. WIREs Climate Change 2, 931-934. http://doi.org/10.1002/wcc.145.

Allison, D., Brown, A.W., George, B.J., Kaiser, K.A. 2016. Mistakes in peer-reviewed papers are easy to find but hard to fix. Nature 530, 27-29. http://doi.org/10.1038/530027a.

Anderegg, W.R.L., Prall, J.W., Harold, J., Schneider, S.H. 2010. Expert credibility in climate change. Proceedings of the National Academy of Sciences 107, 12107-12109.

Aarstad, J. 2010. Expert credibility and truth. Proceedings of the National Academy of Sciences 107, 47. http://doi.org/10.1073/pnas.1012156107.

Bast, J.L. 2013. AMS Survey shows no consensus on Global Warming. Policy Brief. The Heartland Institute, November, 28.

Bedford, D. 2010. Agnotology as a teaching tool: Learning climate science by studying misinformation. Journal of Geography 109, 159-165. http://doi.org/10.1080/00221341.201 0.498121 .

Bedford, D., Cook, J. 2013. Agnotology, scientific consensus, and the teaching and learning of climate change: A response to Legates, Soon and Briggs. Science \& Education 22, 2019 2030 . 
Benestad, R.E., Hygen, H.P., Dorland, R. van, Cook, J., Nuccitelli, D. 2013. Agnotology: learning from mistakes. Earth Science Dynamics Discussion 4, 451-505. https://doi.org/10.5194/ esdd-4-451-2013.

Biddle, J.B., Leuschner, A. 2015 Climate skepticism and the manufacture of doubt: can dissent in science be epistemically detrimental? European Journal for Philosophy of Science 5, 261278.

Bodestein, L. 2010. Regarding Anderegg et al. and climate change credibility. Proceedings of the National Academy of Sciences 107, 52. http://doi.org/10.1073/pnas.1013268108.

Bray, D., von Storch, H. 2013. A survey of the perceptions of climate scientists 2013. Helmholtz Zentrum Geesthacht Geestacht, Germany.

Carlton, J.S., Perry-Hill, R., Huber, M., Prokopy, L.S. 2015. The climate change consensus extends beyond climate scientists. Environmental Research Letters 10, 094025. http://doi. org/10.1088/1784-9326/10/9/094025.

Christy, J.R. 2016. U.S. House Committee on Science, Space \& Technology. 2 Feb 2016. http://docs.house.gov/meetings/SY/SY00/20160202/104399/HHRG-114-SY00-W stateChristyJ-20160202.pdf.

Cook, J., Nuccitelli, D., Green, S.A., Richardson, M., Winkler, B., Painting, R., Way, R., Jacobs, P., Skuce, A. 2013. Quantifying the consensus on anthropogenic global warming in the scientific literature. Environmental Research Letters 8, 024024. http://doi.org/10.1088/17489326/8/2/024024.

Cook, J., Nuccitelli, D., Skuce, A., Jacobs, P., Painting, R., Honeycutt, R., Green, S.A., Lewandowsky, S., Richardson, M., Way, R.G. 2014. Reply to Quantifying the consensus on anthropogenic global warming in the scientific literature: a re-analysis. Energy Policy 73, 706-708. https://doi.org/10.1016/j.enpol.2014.06.002.

Cook, J., Cowtan, K. 2015. Reply to Comment on 'Quantifying the consensus on anthropogenic global warming in the scientific literature'. Environmental Research Letters 10, 039002.

Cook, J., Oreskes, N., Doran, P., Anderegg, W.R.L., Verheggen, B., Maibach, E.W., Carlton, J.S., Lewandowsky, S., Skuce, A.G., Green, S.A., Nuccitelli, D., Jacobs, P., Richardson, M., Winkler, B., Painting, R., Rice, K. 2016. Consensus on Consensus: A Synthesis of Consensus Estimates on Human- caused Global Warming. Environmental Research Letters 11, 048002.

Curry, J. 2011. Nullifying the climate null hypothesis. WIREs Climate Change 2, 919-924. http:// doi.org/10.1002/wcc.141.

Curry, J. 2015. State of the climate debate in the U.S. Remarks to U.K. House of Lords. 15 Junio. https://judithcurry.com/2015/06/15/state-of-the-climate-debate-in-the-u-s/.

Curry, J. 2017. Climate models for the layman. The Global Warming Policy Fundation, Briefing 24. https://www.thegwpf.org/content/uploads/2017/02/Curry-2017.pdf.

Dean, B.J.F. 2015. Comment on 'Quantifying the consensus on anthropogenic global warming in the scientific literature' Environmental Research Letters 10039001.

Doran, P.T., Zimmerman, M.K. 2009. Examining the scientific consensus on climate change. EOS 90, 22-23. http://doi.org/10.1029/2009EO030002.

Duarte, J.L. 2014. Comment on 'scientists' views about attribution of global warming' Enviromental. Science Technology 48, 14057-14058. http://doi.org/10.1021/es504574v.

Engqvist, L., Frommen, J.G. 2008. The h-index and self citation. Trends in Ecology and Evolution 23, 250-252. http://doi.org/10.1016/j.tree.2008.01.009.

Friends of Science Society. 2014. 97\% Consensus? No! Global warming math, myth \& social proofs. Friends of Science Society, Calgary, Canadá, 50 pp. Disponible en: https://www. friendsofscience.org/assets/documents/97_Consensus_Myth.pdf.

Granqvist, R. 2009. Comment on "examining the scientific consensus on climate change". EOS 90, 27. http://doi.org/10.1029/2009EO270008. 
Harari, Y.N. 2016. Homo Deus. Breve historia del mañana. Ed Debate, ISBN: 9788499924212.

Harlos, C., Edgell, T.C., Hollander, J. 2017. No evidence of publication bias in climate change science. Climatic Change 140, 375-385. http://doi.org/10.1007/s 10584-016-1880-1.

Harde, H. (2014). Advanced two-layer climate model for the assessment of global warming by $\mathrm{CO}_{2}$. Open Journal of Atmospheric and Climate Change 1, 2374-3794. http://doi.org/10.15764/ ACC.2014.03001.

Helsdon, J. 2009. Further comment on "Examining the scientific consensus on climate change". EOS 90, 27. http://doi.org/10.1029/2009EO270009.

Houston, J.R., Dean, R.G. 2011. Sea-level acceleration based on U.S. tide gauges and extensions of previous global-gauge analyses. Journal of Coastal Research 27, 409-417. https://doi. org/10.2112/JCOASTRES-D-10-00157.1.

Idso, C.D., Carter, R.M., Singer, S.F. 2015. Why scientists disagree about global warming. The Heartland Institute Arlington Heights, Illinois, $122 \mathrm{pp}$.

IPCC. 2013. Summary for policymakers. In: Climate Change 2013: The physical science basis. In: T.F. Stocker et al. (Eds.) Contribution of Working Group I to the Fifth Assessment Report of the IPCC. Cambridge University Press, Cambridge.

Kahan, D.M., Jenkins-Smith, H, Braman, D. 2011. Cultural cognition of scientific consensus. Journal of Risk Research 14, 147-174. http://doi.org/10.1080/13669877.2010.511246.

Kahan, D.M. 2015. Climate-science communication and the measurement problem. Political Psychology 36, 1-43. http://doi.org/10.1111/pops.12244.

Lafambroise, D. 2016. Peer review. Why skepticism is essential. The Global Warming Policy Foundation Report 20. ISBN 978-0-9931189-3-7.

Lainer, L. (Dir.). 2015. An elaboration of AAAS Scientific's views. A deeper examination of views about key science topics by members of the American Association for Advance Science. Pew Research Center, 48 pp. Disponible en: http://www.pewinternet.org/packages/science-andsociety/.

Legates, D.R., Soon, W., Briggs, W.M., Monckton of Brenchley, C. 2013a. Learning and teaching climate science: the perils of consensus knowledge using agnotology. Science \& Education 22, 2007-2017. http://doi.org/10.1007/s11191-013-95883.

Legates, D.R., Soon, W., Briggs, W.M., Monckton of Brenchley, C. 2013b. Climate consensus and 'misinformation': a rejoinder to agnotology, scientific consensus, and the teaching and learning of climate change. Science \& Education 24, 299-318. http://doi.org/10.1007/ s11191-013-9647-9.

Lefsrud, L.M., Meyer, R.E. 2012. Science or Science Fiction? Professionals' discursive construction of climate change. Organization Studies 33, 1477-1506. http://doi. org/10.1177/0170840612463317.

Lewandowsky, S., Oberauer, K., Gignac, G.E. 2013. NASA Faked the Moon landing-Therefore, (climate) science is a hoax: An anatomy of the motivated rejection of science. Psychological Science 245, 622-633. http://doi.org/10.1177/0956797612457686.

Lewandowsky, S., Cook, J., Lloyd, E. 2016. The 'Alice in Wonderland' mechanics of the rejection of (climate) science: simulating coherence by conspiracism. Synthese. http://doi.org/10.1007/ s11229-016-1198-6.

Lindzen, R. 2012. Climate Science: Is it currently designed to answer questions? Euresis 2, 161193.

Liu, X., Vedlitz, A., Stoutenborough, J.W., Robinson, S. 2015. Scientists' views and positions on global warming and climate change: A content analysis of congressional testimonies. Climatic Change 131, 487-503. http://doi.org/10.1007/s10584-015-1390-6.

Losh, S.C. 2016. Agreement among environmental scientists: higher than previously thought. Bulletin of Science, Technology \& Society 1-6. http://doi.org/10.1177/0270467616634958. 
Lovelock, J. 2008. La venganza de la Tierra. Planeta, 268 pp.

Luening, S., Vahrenholt, F. 2017. Medieval Warning Project Map. Disponible en: http://t1p.de/ mwpdownloaded (consulta 27/08/2017).

Maibach, E., Stenhouse, N., Cobb, S., Ban, R., Bleistein, A. et al. 2012. American Meteorological Society member survey on global warming: Preliminary findings (February 12). Fairfax, VA: Center for Climate Change Communication. Disponible en : http://climate.gmu.edu.

Merton, R.K. 1968. The Matthew effect in science. Science 159, 56-63. http://doi.org/10.1126/ science.159.3810.56.

O’Neil, S.J., Boykoff, M. 2010. Climate denier, skeptic, or contrarian? PNAS 107,39 E1S1 . http:// doi.org/10.1073/pnas.1010507107.

Oreskes, N. 2004. Beyond the ivory tower: the scientific consensus on climate change. Science 306, 5702. http://doi.org/10.1126/science.1103618.

Peiser, B. 2005. The letter science magazine rejected. Energy and Environment 16, 685-688. http:// doi.org/10.1260/0958305054672330.

Petit, J.R., Jouzel, J., Raynaud, D., Barkov, N.I., Barnola, J.M., Basile, I., Bender, M., Chappellaz, J., Davis, M., Delaygue, G., Delmotte, M., Kotlyakov, V.M., Legrand, M., Lipenkov, V.Y., Lorius, C., Pépin, L., Ritz, C., Saltzman, E., Stievenard, M. 1999. Climate and atmospheric history of the past 420,000 years from the Vostok ice core, Antarctica. Nature 399, 429-436. http://doi.org/10.1038/20859.

Pielke, R. Sr., Beven, K., Brasseur, G., Calvert, J., Chahine, M., Dickerson, R.R., Entekhabi, D., Foufoula-Georgiou, E., Gupta, H., Gupta, V., Krajewski, W., Krider, E.P., Lau, W.K.M., McDonnell, J., Rossow, W., Schaake, J., Smith, J., Sorooshian, S., Wood, E. 2009. Climate Change: The need to consider human forcings besides greenhouse gases. EOS 90, 10. http:// doi.org/10.1029/2009EO450008.

Powell, J. 2014. Climate Scientists Virtually Unanimous: Anthropogenic Global Warming Is True. Bulletin of Science, Technology \& Society 1-6. http://doi.org/10.1177/0270467616634958.

Pew Research Center (PRC) (2015) An elaboration of AAAS Scientists 'views. A deeper examination of views about key science topics by members of the American Association for the advancement of Science. In: L. Rainie (Dir.), 48 pp. Disponible en: http://www. pewinternet.org/packages/science-and-society/.

Rapp, D. 2014. Assessing Climate Change. Springer, $3^{\text {a }}$ ed., 816 pp.

Rosenberg, S., Vedlitz, A., Cowman, D., Zahran, S. 2010. Climate Change: A Profile of U.S. Climate Scientists' Perspectives. Institute for Science, Technology and Public Policy, Bush School of Government and Public Service, Texas A\&M University, 20 p. (Consultado en su version original, publicado posteriormente en Climatic Change 101, 311-329).

Schulte, K-M. 2008. Scientific consensus on climate change? Energy \& Environment 19, 281-286.

Stenhouse, N., Maibach, E., Cobb, S., Ban, R., Bleistein, A., Croft, P., Bierly, E., Seitter, K., Rasmussen, G., Leiserowitz, A. 2014. Meteorologists' views about global warming: A survey of American Meteorological Society professional members. Bulletin of the American Meteorological Society 95, 1029-1040. https://doi.org/10.1175.BAMS-D-13-00091.1.

Strengers, B., Verheggen, B., Vringer, K. 2015. Climate science survey. Questions and responses. PBL Netherlands Environmental Assessment Agency, 39 p.

Tol, R.S.J. 2014a. Quantifying the consensus on anthropogenic global warming in the literature: a re-analysis. Energy Policy 73, 701-705.

Tol, R.S.J. 2014b. Quantifying the consensus on anthropogenic global warming in the literature: rejoinder. Energy Policy 73, 709. https://doi.org/10.1016/j.enpol.2014.04.045.

Tol, R.S.J. 2016. Comment on 'Quantifying the consensus on anthropogenic global warming in the scientific literature'. Environmental Research Letters 11, 048001. 
Trenberth, K.E. 2011. Attribution of climate variations and trends to human influences and natural variability. WIREs Climate Change 2, 925-930. http://doi.org/10.1002/wcc.142.

Verheggen, B., Strengers, B., Cook, J. van Dorland, R., Vringer, K., Peters, J. Visser, H., Meyer, L. 2014. Scientists' views about attribution of global warming. Environmental Science \& Technology 48, 8963-8971. http://doi.org/10.1021/es501998e. 